\title{
Qualidade de vida em mulheres mastectomizadas
}

\author{
Quality of life in mastectomized women
}

Aline Raquel Brito Paiva ${ }^{1}$, Cinara Regina Aragão Vieira Monteiro²

\begin{abstract}
Resumo: Introdução: O câncer de mama é a neoplasia de maior incidência entre as mulheres e com alto índice de mortalidade. A mastectomia é uma abordagem terapêutica cirúrgica eficaz, porém pode proporcionar sequelas na qualidade de vida nas pacientes. Objetivo: Avaliar qualidade de vida em mulheres mastectomizadas. Materiais e Métodos: Foi aplicado um questionário em pacientes atendidas em um hospital de referência em oncologia, contemplando dados clínicos e sócio-demográficos e o SF-36 para avaliação da qualidade de vida. O estudo foi aprovado pelo comitê de ética da Universidade Ceuma. Resultados: A faixa etária mais acometida foi de 51 a 60 anos $(32,5 \%)$, mulheres de raça branca $(47,5 \%)$, com grau de escolaridade de nível médio (45\%) e profissionais da lavoura (25\%), acompanhadas no setor ambulatorial do hospital $(97,5 \%)$. O lado mamário esquerdo foi o mais acometido (40\%) e o tratamento mais realizado foi a associação da quimioterapia com a cirurgia $(55 \%)$. O domínio de qualidade de vida mais comprometido foi o de limitação por aspectos emocionais associado ao tipo de cirurgia, em particular a mastectomia total $(p<0,05)$. Conclusão: Tais resultados reforçam a necessidade de uma reabilitação globalizada das pacientes no pós-operatório de mastectomização.
\end{abstract}

Palavras-chave: Câncer de mama, mastectomia, qualidade de vida.

Abstract: Introduction: Breast cancer is the most prevalent neoplasm among women and with a high mortality rate. Mastectomy is an effective surgical therapeutic approach, but may provide sequelae in patients' quality of life. Objective: To evaluate quality of life in mastectomized women. Materials and Methods: A questionnaire was applied in patients attending a reference hospital in oncology, including clinical and socio-demographic data and the SF-36 to evaluate the quality of life. The ethics committee of the Ceuma University approved the study. Results: The most affected age group was 51 to 60 years old $(32.5 \%)$, white women $(47.5 \%)$, medium level of education (45\%) and professionals in the field (25\%), accompanied in the outpatient sector of the hospital (97.5\%). The left breast side was the most affected $(40 \%)$ and the most used treatment was the association of chemotherapy with surgery (55\%). The domain of the most compromised quality of life was the limitation for emotional aspects associated with the surgery type, in particular the total mastectomy $(p<0.05)$. Conclusion: These results reinforce the need for a globalized rehabilitation of patients in the postoperative period of mastectomization.

Key words: Breast cancer, mastectomy, quality of life.

1- Discente do curso de Fisioterapia - Universidade CEUMA, São Luís - Maranhão, Brasil.

2- Preceptora de estágio hospitalar do curso de Fisioterapia - Universidade CEUMA, São Luís - Maranhão, Brasil. 


\section{Introdução}

O câncer é um grande problema de saúde pública no mundo. A neoplasia maligna de mama é a mais prevalente entre as mulheres. Nos Estados Unidos da América (EUA), essa enfermidade é a mais comum entre as mulheres, estima-se 61.000 novos casos de carcinoma de mama feminino, e juntamente com os cânceres de pulmão e cólon retal correspondem a $46 \%$ de causa de morte por câncer ${ }^{6}$. No Brasil, a neoplasia mamária é considerada a mais prevalente, principalmente entre mulheres de 40 e 69 anos. De acordo com o Instituto Nacional do Carcinoma (INCA), nos estados brasileiros, esta doença possui grande representatividade, onde a região Sudeste se destaca com 65 novos casos por 100.000 mulheres/ano ${ }^{11}$.

Os fatores de riscos mais comuns são a idade, histórico familiar, e modificações endócrinas, comportamentais, ambientais, genéticas e hereditárias. A vida reprodutiva tem sido intimamente relacionada à incidência de casos da doença, evidências consistentes apontam que a nuliparidade, terapia hormonal na menopausa e período prolongado entre a menarca e o primeiro parto são fortes condições de risco. A amamentação consiste em um fator protetor para o câncer mamário ${ }^{1}$.

As modalidades terapêuticas incluem quimioterapia, radioterapia, cirurgia e hormonioterapia. A mastectomia se caracteriza pela retirada parcial ou total da mama, a depender da necessidade e comprometimento. O tratamento cirúrgico pode ser complementado pelas outras definição terapêutica é determinada pela possibilidade do tumor em responder ao tratamento e ao aumento da sobrevida. As limitações funcionais e emocionais decorrentes da mastectomização interferem na qualidade de vida das mulheres ${ }^{15}$.

A mulher portadora de câncer de mama evolui ao pensamento relacionado a morte ou com desfecho infeliz, que passa a ter alterações do seu papel feminino na sociedade com sofrimento por pela perda de parte do seu corpo, desencadeando doenças futuras como depressão, ansiedade e o medo exacerbado ${ }^{3}$.

A reabilitação dessas alterações depende da extensão da área atingida e da gravidade da doença, porém visa essencialmente recuperar as funções comprometidas, prolongar a sobrevida e melhorar a qualidade de vida dessas pacientes em todas as etapas do tratamento oncológico 7, 2, 12, 10. Em cada etapa, a conduta fisioterapêutica deve ser traçada com orientações domiciliares e tratamentos específicos para recuperação funcional, evitar aderência cicatricial e minimizar o linfedema ${ }^{2,8}$.

Este estudo objetivou avaliar a qualidade de vida das mulheres mastectomizadas e contribuiu para identificar quais aspectos são mais afetados pelo procedimento cirúrgico que interferem na manutenção da funcionalidade.

\section{Materiais e Métodos}

$\begin{gathered}\text { Trata-se de um estudo } \\ \text { qualitativo, }\end{gathered}$
epidemiológico, observacional e
descritivo,
transversal, realizada com um grupo
de 40 mulheres, mastectomizadas,


com idade igual e/ou a superior a 18 anos, que aceitaram participar da pesquisa assinando 0 termo de consentimento livre e esclarecido (TCLE), atendidas e acompanhadas no Hospital Tarquínio Lopes Filho, em São Luís - Maranhão.

A coleta dos dados foi feita através da aplicação de um questionário, preenchido parcialmente pela pesquisadora e parcialmente pelas pacientes, porém sob supervisão da pesquisadora. $O$ questionário contempla dados sóciodemográficos, dados clínicos e SF 36 desenvolvido em 1992 por Ware e Sherbourne e validado no Brasil por Ciconelli et al. (2011) para avaliação da qualidade de vida. Os dados em que o paciente não soube responder foram colhidos através dos prontuários disponíveis nas enfermarias ou setor de quimioterapia. A avaliação da qualidade de vida contempla 8 domínios: dor, saúde mental, vitalidade, aspectos físicos, capacidade funcional, estado geral, aspectos sociais e aspectos emocionais.

Os dados foram avaliados pelo programa NCSS 11 (2016). Inicialmente foi feito a estatística descritiva de todos as variáveis através de gráficos e tabelas de frequências. Com estimativas de média, desvio-padrão, máximo e mínimo das variáveis numéricas e das variáveis ordinais (qualidade de vida) estimou-se as medianas e o intervalo interquartil. Posteriormente, para se avaliar associação das variáveis clínicas e qualidade de vida. Para avaliar a correlação foi realizado o teste não paramétrico de Spearman. O nível de significância para se rejeitar a hipótese de nulidade será de $5 \%$, ou seja, considerar-se-á como estatisticamente significativo um valor de $p<0,05$.

Esta pesquisa foi aprovada pelo comitê de ética da Universidade CEUMA, através do parecer № 2.145.270.

\section{Resultados}

A amostra da pesquisa foi composta por 40 pessoas mulheres, mastectomizadas, assistidas no Hospital de Tarquínio Lopes Filho, referência oncológica no estado. As características sócio - demográficas estão demonstradas na Tabela 1.

As características clínicas sobre o tipo de câncer, tempo de doença, presença e localização de metástase, e tipo de tratamento e cirurgia realizados estão descritos na tabela 2.

A avaliação da qualidade de vida evidenciou que a capacidade funcional, limitação por aspectos físicos e emocionais foram os domínios mais afetados neste grupo de mulheres mastectomizadas como demonstrado na tabela 3.

A limitação por aspectos emocionais demonstrou associação quanto ao tipo de procedimento cirúrgico onde a mastectomia total apresentou maior comprometimento deste domínio $(p=0,026)$. 
Tabela 1: Dados sócio - demográficos de mulheres mastectomizadas

\begin{tabular}{lcc}
\hline \multicolumn{1}{c}{ Variáveis } & $\mathbf{n}$ & $\%$ \\
\hline Idade & & \\
$<31$ & 5 & 12,5 \\
$31-40$ & 10 & 25,0 \\
$41-50$ & 6 & 15,0 \\
$51-60$ & 13 & 32,5 \\
$>60$ & 6 & 15,0
\end{tabular}

Cor/Raça

Branca

$19 \quad 47,5$

Parda

$10 \quad 25,0$

Negra

$11 \quad 27,5$

Escolaridade

Analfabeto

25,0

Ens. Fundam. Incompleto

$9 \quad 22,5$

Ens. Médio Completo

$18 \quad 45,0$

Ens. Superior Completo

$11 \quad 27,5$

Atividade profissional

\begin{tabular}{lcc} 
Lavradora & 10 & 25,0 \\
Dona de casa & 8 & 20,0 \\
Pedagoga & 5 & 12,5 \\
Doméstica & 4 & 10,0 \\
Enfermeira & 2 & 5,0 \\
Outros & 11 & 27,5 \\
Procedência & & \\
Ambulatorial & 39 & 97,5 \\
Internação & 1 & 2,5 \\
\hline \multicolumn{1}{c}{ Total } & 40 & 100,0 \\
\hline
\end{tabular}


Tabela 2: Dados clínicos sobre o câncer mamário das pacientes mastectomizadas.

\begin{tabular}{lcc}
\hline \multicolumn{1}{c}{ Variáveis } & n & $\%$ \\
\hline Tipo de câncer & & \\
Direito & 15 & 37,5 \\
Esquerdo & 16 & 40,0 \\
Bilateral & 8 & 20,0 \\
Ignorado & 1 & 2,5
\end{tabular}

\section{Tempo da doença (anos)}

$\begin{array}{lcc}<1 & 2 & 5,0 \\ 1 & 13 & 32,5 \\ 1-2 & 16 & 40,0 \\ 2-3 & 4 & 10,0 \\ >3 & 5 & 12,5\end{array}$

\section{Metástase}

Sim

$19 \quad 47,5$

Não

1947,5

Ignorado

25,0

\section{Localização}

Não especificou

$21 \quad 52,5$

Pulmão

$13 \quad 32,5$

Fígado

25,0

Coluna

$1 \quad 2,5$

Crânio

$1 \quad 2,5$

Olhos

$1 \quad 2,5$

Ossos

$1 \quad 2,5$

Tipo de tratamento

Quimioterapia e Cirurgia

$22 \quad 55,0$

Quimioterapia, Radioterapia e

Cirurgia

$18 \quad 45,0$

Tipo de cirurgia

Mastectomia total

Quadrantectomia

Mastectomia total e

Quadrantectomia

$$
\text { Total }
$$

\begin{tabular}{cc}
19 & 47,5 \\
17 & 42,5 \\
4 & 10,0 \\
\hline 40 & 100,0 \\
\hline
\end{tabular}


Tabela 3: Dados da avaliação da qualidade de vida da amostra estudada.

\begin{tabular}{lccccc}
\hline \multicolumn{1}{c}{ Variáveis } & N & Mínimo & Máximo & Média & $\begin{array}{c}\text { Desvio } \\
\text { Padrão }\end{array}$ \\
\hline Capacidade Funcional & 40 & 0 & 100 & 32,00 & 29,41 \\
Saúde Mental & 40 & 0 & 100 & 56,10 & 28,26 \\
Limitação Física & 40 & 0 & 75 & 4,38 & 16,88 \\
Limitação Emocional & 40 & 0 & 100 & 27,25 & 18,23 \\
Aspectos Sociais & 40 & 12 & 100 & 64,08 & 25,40 \\
Dor & 40 & 10 & 100 & 61,40 & 24,44 \\
Estado Geral de Saúde & 40 & 5 & 97 & 62,13 & 23,75 \\
Vitalidade & 40 & 0 & 100 & 53,00 & 24,38 \\
\hline
\end{tabular}

\section{Discussão}

As principais alterações encontradas nesse estudo em relação aos domínios da qualidade de vida com maior comprometimento foram limitação por aspectos físicos, limitação por aspectos emocionais e a capacidade funcional.

Dor no ombro, diminuição da ADM, dificuldade na realização de exercícios físicos e/ou atividades diárias, câimbra no membro superior homolateral à cirurgia, diminuição da força muscular com diminuição para os movimentos de abdução, flexão e rotação lateral do ombro homolateral foram observados em pacientes mastectomizadas, corroborando com os resultados do estudo realizado reforçando o aparecimento de sequelas da motricidade $e$ funcional após a cirurgia. $\mathrm{Na}$ avaliação da qualidade de vida (SF 36) foi demonstrado maior comprometimento nos domínios de limitação por aspectos físicos, vitalidade e dor 13,16 .

A aplicação de um protocolo de exercícios em um grupo de mulheres submetidas ao tratamento oncológico mamário resultou em melhora nos aspectos clínicos e funcionais, evitando declínio nos domínios da qualidade de vida ${ }^{14}$.
A vida amorosa, atividade laboral e situação econômica de mulheres jovens mastectomizadas sofreram impacto negativo após a cirurgia. A médio e longo prazo houve declínio significativo do estado geral de saúde, aspectos físicos, capacidade funcional e surgimento de dor. O câncer de mama e seu tratamento provocam alterações e limitações que afetam significativamente a vida das pacientes a curto, médio e longo prazo ${ }^{5}$.

O câncer de mama é o câncer mais temido entre as mulheres, pelo fato do trauma psicológico que a doença desenvolve, quanto ao tratamento e ao medo da mutilação e distorção da autoimagem, comprometendo o aspecto físico, psicológico e social; pois a mama apresenta grande importância para o corpo da mulher como parte simbólica e característica da imagem feminina, faz relação com a sexualidade e também com a função de mulher na sociedade ${ }^{9}$.

\section{Conclusão}

Após a realização do estudo percebeu-se a importância da assistência multidisciplinar global no acompanhamento pós-operatório de 
mulheres mastectomizadas, com ênfase na reabilitação. A mastectomia acarreta diversas alterações nos aspectos psicológicos, sexuais, laborais, sociais e funcionais, é importante que essas alterações sejam identificadas precocemente para prevenção de complicações futuras que possam comprometer a qualidade de vida das mulheres submetidas à cirurgia de mama.

Agradecimentos: Primeiramente a Deus por ter permitido que chegasse até aqui com saúde e força para superar as dificuldades, aos meus pais por ter me incentivado e concedido os estudos, a minha orientadora Cinara Aragão por tanto zelo, dedicação e confiança para elaboração deste trabalho e por fim as minhas amigas Taise e Hellen que estiveram comigo nessa jornada, vocês com certeza fazem parte dessa vitória.

Conflito de interesses: nada a declarar.

\section{Referências}

1. Anderson KN, Schwab RB, Martinez ME. Reproductive risk factors and breast cancer subtypes: a review of the literature. Breast Cancer Res Treat, 2014; 144:1-10

2. Bergmann A, Ribeiro MIP, Pedrosa E, Nogueira EA, Oliveira ACG. Fisioterapia em mastologia oncológica: rotinas do Hospital do Câncer III/INCA. Rev Bras Cancerol 2006; 52:97-109.

3. Caetano, AJ. Soares, E. Mulheres mastectomizadas diante do processo de adaptação do self-físico e self- pessoal. Revista Enfermagem 2005; 210-216.

4. Ciconelli RM, Campolina AG, Bortoluzzo AB, Ferraz MB. Validação da versão brasileira do questionário genérico de qualidade de vida shortform 6 dimensions (SF -6D Brasil). Ciência e Saúde Coletiva 2011; 16:3103-3110.

5. Conde CR, Lemos TMR, Pozati MPS, Ferreira MLSM. A repercussão do diagnóstico e tratamento do câncer de mama no contexto familiar. Investigação Qualitativa em Saúde//Investigación Cualitativa en salud//Atas CIAIQ 2016; 2:15441553.

6. DeSantis CE, MA J, Sauer AG, Newman LA, Jemal A. Breast Cancer Statistics, 2017, Racial Disparity in Mortality by State. Ca Cancer J Clin, 2017; 67:439-448.

7. Duarte TP, Andrade AN. Enfrentando a Mastectomia: análise dos relatos de mulheres mastectomizadas sobre questões ligadas à sexualidade. Estudos de Psicologia 2006; 8:1551635.

8. Fabro EAN, Costa RM, Oliveira JF, Lou MBA, Torres DM, Ferreira FO, Macedo FO, Carvalho CM, Ribeiro MJP, Bergman A. Atenção fisioterapêutica no controle do linfedema secundário ao tratamento do câncer de mama: rotina do Hospital do Câncer III/Instituto Nacional do Câncer. Revista Brasileira Mastologia, 2016; 26:4-8.

9. Gouveia PF, Gonzalez EO, Grer PA, Fernades Ca, Lima MC. Avaliação da amplitude de movimento e força da cintura escapular em pacientes de pós-operatório tardio de mastectomia radical modificada. Revista Fisioterapia e Pesquisa 2008;15:172-6. 
10. Grant A, Walton SI, Knapik G, Collacutt VI, Brigden M. Rehabilitative medicine and physiotherapy services in community oncology. Feature 2017, 16:10-15.

11. INCA. Instituto Nacional de Câncer. Câncer de mama: é preciso falar disso. / Instituto Nacional de Câncer José Alencar Gomes da Silva. - 4. ed. - Rio de Janeiro: Inca, 2016.

12. Kabak VY, Tas N, Ekinci $Y$, Uysal $S A$, Düger $T$. Investigation on The Physical and Functional Needs in Adult Cancer Patients Consulted to Physioterapy and Rehabilitation. Turkish Journal of Oncology 2016; 31:104-108.

13. Lahoz MA, Nyssen SM, Correia GNn, Garcia Apu, Driusso P. Capacidade Funcional e Qualidade de Vida em Mulheres Pós Mastectomizadas. Revista Brasileira de Cancerologia 2010; 56:423-430.

14. Leites GT, Knorst MR, Lima CHLI, Zerwes FP, Frison VP. Fisioterapia em oncologia mamária: qualidade de vida e evolução clínico funcional. Revista Ciência \& Saúde 2010; 3:14-21.

15. Silva CB, Albuquerque V, Leite J. Qualidade de vida em pacientes portadoras de neoplasia mamária submetidas a tratamentos quimioterápicos. Revista Brasileira Cancerologia 2010; 56:227-36.

16. Vieira RAC, Silva FCB, Biller G, Silva JJ, Paiva CE, Sarri AJ. Instrumentos de avaliação quantitativa e qualitativa das sequelas relacionadas ao tratamento de câncer de mama. Revista Brasileira Mastologia 2016; 26:126-132. 